\title{
Mechanical Simulations Regarding the Influence of Paper Insulation Degradation on the Radial Mechanical Strength of Continuously Transposed Conductors for Power Transformers
}

\author{
Dipl.-Ing. D. Geißler (daniel.geissler@kit.edu), Prof. Dr.-Ing. T. Leibfried \\ Institute of Electric Energy Systems and High Voltage Technology \\ Karlsruhe Institute of Technology (KIT), Karlsruhe, Federal Republic of Germany
}

\begin{abstract}
The short circuit withstand capability of power transformers describes the ability of the windings to withstand electromagnetic forces resulting from current density in the conductors and magnetic stray fields. Especially operationally aged transformers often have a reduced short circuit strength what can lead to irreversible deformation of windings if high fault currents occur. Short circuit strength is to some degree dependent on the mechanical strength of the conductors. Mainly the cellulosic insulation materials experience degradation during operation time due to high temperatures and moisture. Hence a reduction in short circuit strength can be correlated to some degree with the aging of the cellulose. In this paper the influence of paper insulation around Continuously Transposed Conductors (CTC) on their mechanical strength will be analyzed by a simplified analytical model as well as by a FEA based mechanical simulation model.
\end{abstract}

\section{Introduction}

CTC deliver an important contribution for the increase of power transformers' efficiency compared to the conventional paper-insulated solid conductors. CTC based windings mainly reduce eddy current losses through their division in single strands. Moreover loop voltages as a result of radial varying magnetic stray fields are compensated by transposition of the strands. Also the winding space factor can be increased as a result of the thin insulation of the single strands. Hence the temperature distribution throughout the whole winding is more homogeneous [1].

Electromagnetic forces can be divided into a radial and axial direction of action. In this paper only radial force components are considered as the rigidity of CTC especially in radial direction is much lower compared to a solid conductor with identical outer dimensions. The reason here for is that in axial direction the cross-section of CTC compared to a solid conductor is only divided into two stacks, while radial direction is divided into usually 10 to 40 single strands.

Radial forces acting on the inner winding are directed inwards resulting in a bending stress. Outward directed forces on the outer winding result in hoop stress. Thus inward directed forces are more critical, as they can lead to buckling of CTC between to axial spacers while outward directed forces only generate a strain of the conductor in azimuthal direction.
The first part of this paper deals with the influence of paper insulation around CTC on the radial bending stiffness with respect of linear material properties: A constant modulus of elasticity for paper and copper and some further simplifying assumptions enable the use of simple equations of bending theory.

The more complex nonlinear case will then be analyzed in the second part by the use of a FEA based model, which describes a CTC segment between two spacers under the influence of radial acting forces. The age condition of paper insulation is respected by varying the corresponding stress strain characteristics in the FEA.

\section{Linear, analytic CTC model}

\subsection{Structure of CTC}

CTC are made of an odd number of rectangular, enamel coated copper strands, which are arranged in two stacks. These two stacks are isolated by a pressboard separator. The number of strands is odd, as always one strand transposes its radial position after a fixed distance in turn direction, called transposition pitch. The whole assembly is isolated by the use of several layers of paper (Fig. 1).
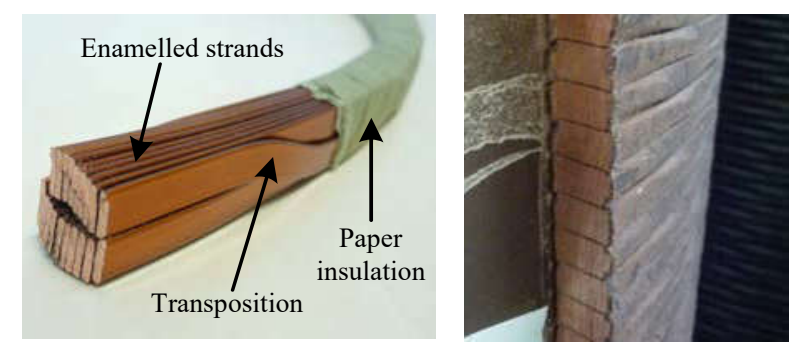

Fig. 1: CTC with partially removed paper insulation (left), cut through a CTC layer winding (right)

The CTC geometric parameters used in this paper are shown in Fig. 2. The strands are described by the axial height $h_{\mathrm{EL}}$ and the radial width $t_{\mathrm{EL}}$. Paper insulation thickness is represented by $d_{\text {Pap }}$. The axial and radial CTC dimensions $H_{\mathrm{CTC}}$ and $T_{\mathrm{CTC}}$ can be calculated as shown in formula (1.1) and (1.2). Here $N_{\mathrm{R}}$ describes the number of strands in radial direction. The separator thickness is neglected.

$$
\begin{aligned}
& H_{\mathrm{CTC}}=H+2 \cdot d_{\text {Pap }}=2 \cdot h_{\mathrm{EL}}+2 \cdot d_{\text {Pap }} \\
& T_{C T C}=T+2 \cdot d_{\text {Pap }}=N_{\mathrm{R}} \cdot t_{\mathrm{EL}}+2 \cdot d_{\text {Pap }}
\end{aligned}
$$




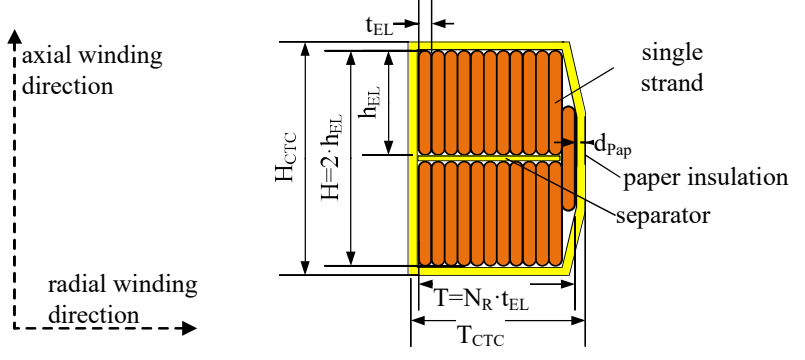

Fig. 2 - CTC cross-section and geometric parameters

\subsection{Radial Bending stiffness $\mathbf{S}_{\mathbf{z}}$}

Bending stiffness is a quantity that describes the ability of a bar-shaped geometry to withstand bending. If the curvature of a CTC segment between two axial spacers is neglected the linear bending theory for a straight beam can be used to describe displacement dependent on applied load and beam geometry. As an approach the electromagnetic force that is distributed over the whole conductor volume will be replaced by a line load $\mathrm{q}$ acting on its surface.

Fig. 3 shows a uniformly loaded beam with simply supported ends. Maximum deflection in the middle of the beam can be calculated by (1.3), [2]. The bending stiffness $S_{z}$ as product of modulus of elasticity $E$ and second moment of inertia $I_{z}$ is the only quantity that contains parameters of the geometry and material of cross-section. As $\mathrm{z}$ is the bending axis, $I_{z}$ can be calculated for a massive cross-section after (1.4)
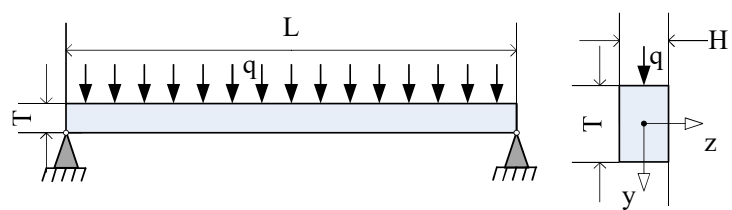

Fig. 3: uniformly loaded beam as approximation of CTC segment between two axial sticks

$$
\begin{gathered}
y_{\max }=\frac{5}{384} \cdot \frac{q \cdot L^{4}}{E \cdot I_{z}}=\frac{5}{384} \cdot \frac{q \cdot L^{4}}{S_{z}} \\
I_{z}=\frac{1}{12} \cdot H \cdot T^{3}
\end{gathered}
$$

To regard influence of paper insulation on the radial mechanical strength it thus is sufficient to focus on the bending stiffness $S_{z}$ of CTC.

The effective bending stiffness $S_{z \text {,eff }}$ for a composite cross-section can be calculated according (1.5) if the single cross-sections are not bonded. In the case of a CTC that means the single strands can move frictionless against each other or against paper insulation.

$$
S_{\mathrm{z}, \mathrm{eff}}=\sum_{i} E_{i} \cdot I_{z, i}
$$

Fig. 4 shows the composite cross-section of a CTC where single paper layers are approximated by rectangular tubes, as the spiral wrapping of insulation paper stripes cannot be respected. Thus in a layer winding without radial spacers the winding's axial clamping force will press all top and bottom CTC paper-layers against each other (Fig. 1). This avoids movement of the single layers against each other under the influence of bending and allows the simplification of rectangular tubes. Furthermore the transposition is neglected.

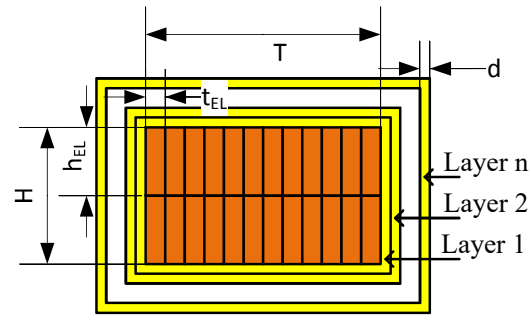

Fig. 4: Simplified cross-section of CTC

The second moment of inertia for a single strand $I_{\mathrm{EL}, Z}$ can be calculated by the use of (1.6).

$$
I_{\mathrm{EL}, \mathrm{z}}=\frac{1}{12} \cdot h_{\mathrm{EL}} \cdot t_{\mathrm{EL}}^{3}
$$

The second moments of inertia for the individual paper layers $I_{\mathrm{Pap}, z, i}$ can be calculated as shown below:

$$
\begin{aligned}
I_{\text {Pap }, z, 1}= & \frac{1}{12} \cdot\left((T+2 d)^{3} \cdot(H+2 d)-T^{3} \cdot H\right) \\
I_{\text {Pap }, z, 2}= & \frac{1}{12} \cdot\left((T+4 d)^{3} \cdot(H+4 d)-(T+2 d)^{3} \cdot(H+2 d)\right) \\
& \vdots \\
I_{\text {Pap }, z, n}= & \frac{1}{12} \cdot\left((T+2 n d)^{3} \cdot(H+2 n d)-\right. \\
& \left.(T+2(n-1) d)^{3} \cdot(H+2(n-1) d)\right)
\end{aligned}
$$

The effective second moment of inertia for $\mathrm{n}$ insulation layers can be summed up according to (1.7). It is shown that paper layers can be summarized to a homogeneous insulating layer with a total thickness $d_{\text {Pap }}=n \cdot d$.

$$
\begin{aligned}
I_{\text {Pap }, z, \text { eff }} & =\sum_{i=1}^{n} I_{\text {Pap }, z, i} \\
& =\frac{1}{12} \cdot\left((T+2 n d)^{3} \cdot(H+2 n d)-T^{3} H\right) \\
& =\frac{1}{12} \cdot\left(\left(T+2 d_{\text {Pap }}\right)^{3} \cdot\left(H+2 d_{\text {Pap }}\right)-T^{3} H\right)
\end{aligned}
$$

So the effective bending stiffness for a CTC with $2 N_{\mathrm{R}}$ strands and $n$ paper layers can be calculated by (1.8).

$$
\begin{aligned}
S_{\mathrm{CTC}, z, \text { eff }} & =S_{\mathrm{Cu}, z \text {,eff }}+S_{\text {Pap }, z \text { eff }} \\
& =E_{\mathrm{Cu}} \cdot I_{\mathrm{Cu}, z, \text { eff }}+E_{\mathrm{Pap}} \cdot I_{\mathrm{Pap}, z \text {,eff }} \\
& =E_{\mathrm{Cu}} \cdot 2 N_{\mathrm{R}} \cdot I_{\mathrm{EL}, z}+E_{\mathrm{Pap}} \cdot I_{\text {Pap }, z, \text { eff }}
\end{aligned}
$$

\subsection{Influence of paper insulation on $\mathbf{S}_{\boldsymbol{z}}$}

To visualize the contribution of paper insulation stiffness to effective CTC bending stiffness the quotient $Z$ according was defined, (1.9).

$$
Z=\frac{S_{\mathrm{CTC}, z, \mathrm{eff}}}{S_{\mathrm{Cu}, z, \mathrm{eff}}}=1+\frac{S_{\mathrm{Pap}, z \text { eff }}}{S_{\mathrm{Cu}, z, \mathrm{eff}}}
$$


4 different CTC geometries with varying radial width T according to Table 1 were examined. Fig. 5 shows the quotient $\mathrm{Z}$ in a $3 \mathrm{D}$ surface plot for these CTC geometries. Therefore paper thickness $d_{\text {Pap }}$ and radial number of strands per stack $N_{\mathrm{r}}$ is varied. It can be seen that especially for a high number of strands in radial direction the influence of paper insulation on effective bending stiffness increases: $Z$ reaches values up to 80 . The reason here for is the smaller radial width $t_{\mathrm{EL}}$ of strands what leads to a significant decrease in radial bending stiffness $S_{\mathrm{Cu}, \mathrm{z}, \mathrm{eff}}$. It is clear that with the increase in total paper thickness $d_{\text {Pap }}$ the paper bending stiffness rises and hence $Z$ rises.

So the simplified analytic consideration proofs an influence of paper on radial bending stiffness of CTC. But the simplifications that were necessary are responsible for a mainly qualitative character of the analytic results.

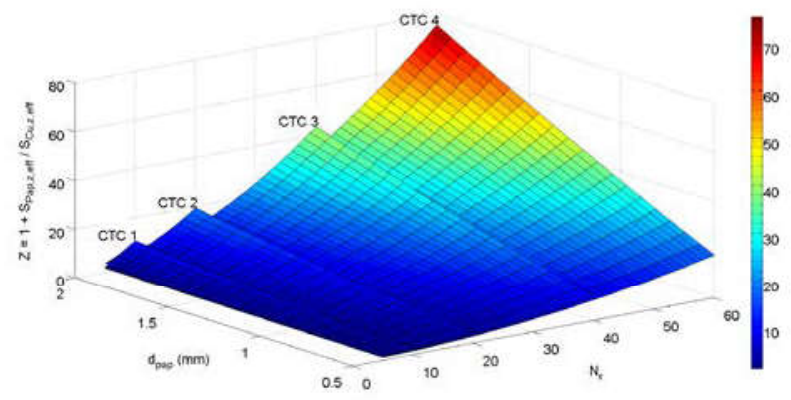

Fig. 5: Visualization of $Z\left(N_{r}, d_{\text {Pap }}\right)$ for 4 CTC's

Table 1: Specification of CTC

\begin{tabular}{rcccc}
\hline & CTC 1 & CTC 2 & CTC 3 & CTC 4 \\
\hline $\mathrm{T}(\mathrm{mm})$ & 10 & 20 & 40 & 60 \\
$\mathrm{~N}_{\mathrm{r}}$ & $5 \ldots 10$ & $5 \ldots 20$ & $5 \ldots 40$ & $5 \ldots 60$ \\
$\mathrm{H}(\mathrm{mm})$ & \multicolumn{4}{c}{15} \\
$\mathrm{~N}_{\mathrm{z}}$ & \multicolumn{5}{c}{2} \\
$\mathrm{~d}_{\text {Pap }}(\mathrm{mm})$ & $0,5 \ldots 2$ \\
$\mathrm{E}_{\mathrm{Cu}}(\mathrm{GPa})$ & \multicolumn{5}{c}{130} \\
$\mathrm{E}_{\text {Pap }}(\mathrm{GPa})$ & \multicolumn{5}{c}{5} \\
\hline
\end{tabular}

\section{FEA based model for CTC}

As the analytic considerations only respect linear material properties and a straight CTC a more detailed model was built by the use of a FEA based mechanical simulation program. But also here some simplifying assumptions have to be accepted: Transposition will still remain neglected to simplify $3 \mathrm{D}$ geometry. They are supposed to have no significant influence on radial bending stiffness of CTC. The spiral paper wrapping is approximated by rectangular tubes of paper as already shown in Fig. 4. Friction and dynamic effects like inertia are neglected.

\subsection{Geometric setup}

The FEA model respects a CTC segment between two axial sticks. To speed up computation time two symmetry planes were introduced to reduce the model's complexity (Fig. 6). The $(r, z)$-plane divides the CTC in the middle between the two axial sticks. The $(r, \varphi)$ plane lies between the two stacks of strands. As only radial forces and thus deformations will be simulated it is sufficient to regard one stack. Table 2 shows geometric parameters for the FEA CTC model (Fig. 7).

Table 2: geometric parameters for the FEA CTC model

\begin{tabular}{lll}
\hline parameter & value & description \\
\hline $\mathrm{h}_{\mathrm{EL}}(\mathrm{mm})$ & 7.65 & axial strand height \\
$\mathrm{t}_{\mathrm{EL}}(\mathrm{mm})$ & 1.22 & radial strand width \\
$\mathrm{N}_{\mathrm{r}}$ & 10 & strands per stack \\
$\alpha_{\mathrm{L}}\left({ }^{\circ}\right)$ & 15 & angle of CTC segment between 2 axial sticks \\
$\mathrm{b}_{\mathrm{L}}(\mathrm{mm})$ & 20 & width of axial spacer in azimuthal direction \\
$\mathrm{t}_{\mathrm{L}}(\mathrm{mm})$ & 15 & radial width of axial spacer \\
$\mathrm{D}_{\mathrm{K}}(\mathrm{mm})$ & 1020 & core outer diameter \\
$\mathrm{N}_{\text {Pap }}$ & 10 & number of paper layers \\
$\mathrm{d}_{\text {Pap }}(\mathrm{mm})$ & 0.6 & total paper thickness \\
\hline
\end{tabular}

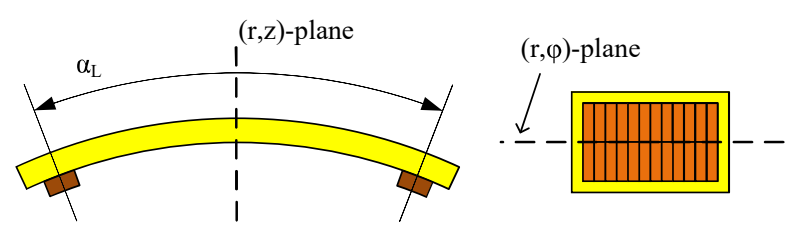

Fig. 6: Symmetry planes

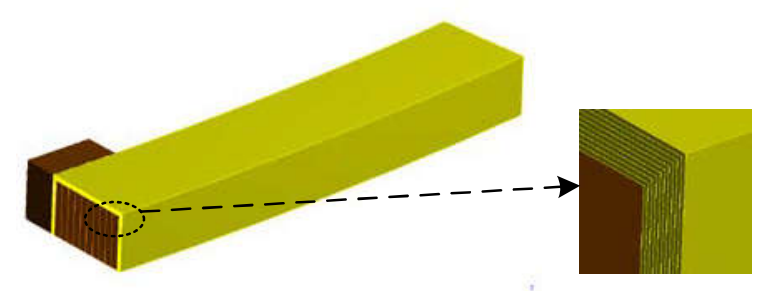

Fig. 7: 3D CTC model

\subsection{Material properties}

The model consists of 3 different materials: The copper strands, the insulating paper and pressboard for the axial spacers. For copper and paper nonlinear stress strain curves were implemented to realize the nonlinear material characteristic under the influence of bending. The spacer is implemented with a linear modulus of elasticity, as it only has the function to support the CTC and no plastic compression will occur. To describe a nonlinear material the FEA Software needs the plastic part of corresponding stress strain characteristic which can be calculated from the data of a tensile test as shown in (1.10) and (1.11) with mechanical stress $\sigma$, the strain $\varepsilon$, and the linear modulus of elasticity $E_{0}$.

$$
\begin{gathered}
\varepsilon_{\mathrm{pl}}=\ln (\varepsilon)-\sigma \cdot(1+\varepsilon) / E_{0} \\
\sigma_{\mathrm{pl}}=\sigma \cdot(1+\varepsilon)
\end{gathered}
$$

Two different sorts of copper were used for simulation with both having a modulus of elasticity of $\mathrm{E}=130 \mathrm{GPa}$ but different plastic strain stress data (Fig. 8 left). "Copper90" has a yield stress of $90 \mathrm{MPa}$, while "Copper150" has a yield stress of $150 \mathrm{MPa}$.

To regard the influence of paper aging state 3 different plastic stress strain curves were used in simulation (Fig. 
8 right). Hereby "aged1" paper has half of the maximum tensile stress compared to the new paper, while "aged2" paper has the same plastic data but a bisected linear modulus of elasticity.
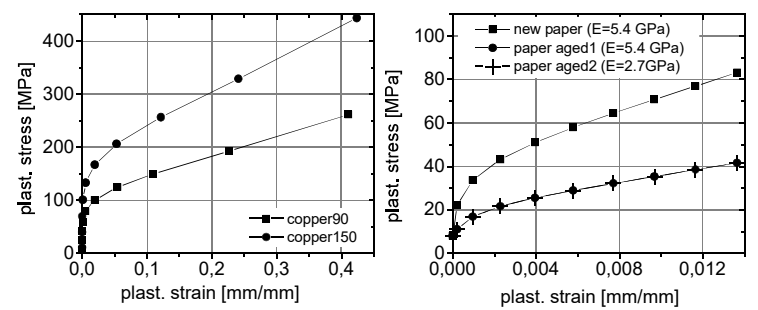

Fig. 8: Plastic stress strain curves for copper (left) and paper (right)

\subsection{Boundary conditions and discretization}

In FEA Software the CTC model had to be fixed by appropriate boundary conditions to gain reasonable simulation results. Thus deformation of the inner face of the axial stick was restricted in all 3 spatial directions what relates to the rigid constraint of the transformer core. All cross sections at the symmetry cut planes had to be restricted in normal respectively azimuthal direction.

As FEA Software only allows excitation by force or pressure on single faces, the radial Lorentz force density $f_{r}$ acting in the complete volume of all copper strands (1.12) has to be approximated. Here for a pressure $p_{\mathrm{EL}}$ acting in inward radial direction on the single strands was chosen. To simulate plastic material behavior with FEA the pressure $p_{\mathrm{EL}}$ has to be applied in the form of a ramp (1.13). Simulation time was set to $1 \mathrm{~s}$ as in a static structural simulation this time has no effect on results.

$$
\begin{gathered}
f_{r}=J_{\varphi} \cdot B_{z}\left[\mathrm{~N} / \mathrm{m}^{3}\right] \\
p_{\mathrm{EL}}(t)=\left(\int_{r_{n}}^{r_{n}+t_{\mathrm{EL}}} f_{r} \cdot d r\right) \cdot \frac{t}{1 \mathrm{~s}}=\hat{p}_{\mathrm{EL}} \cdot \frac{t}{1 \mathrm{~s}}\left[\mathrm{~N} / \mathrm{m}^{2}\right](
\end{gathered}
$$

Discretization of CTC model was done by different types of mesh elements: For the strands quadratic 3D Solid-Elements (Solid186) were used with 2 elements in radial direction. The paper layers were meshed with Solid-Shell Elements (Solsh190) using 1 element in paper thickness direction, Fig. 10. Shell elements are well suited to model thin structures where one dimension is much smaller than the other two dimensions [3].

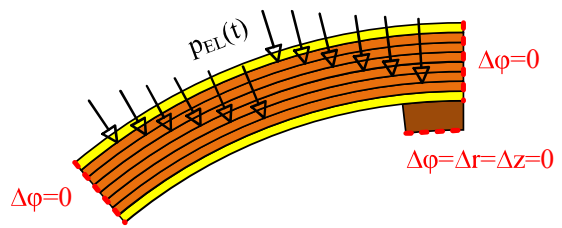

Fig. 9: Boundary conditions and excitation of CTC model

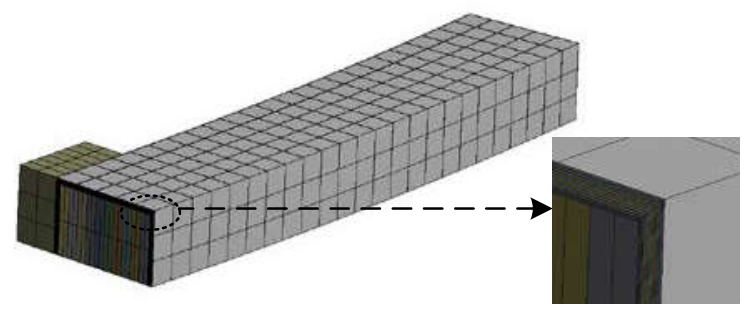

Fig. 10: Meshed CTC model

\section{FEA Simulation results}

Simulations were done for a CTC with geometric parameters according to Table 2. For both copper species "Cu90" and "Cu150" the material property of paper insulation was varied:

First simulation was done without paper to have a reference of the behavior of the "blank" CTC. The following simulations were done with the 3 plastic stress strain curves for the paper insulation according to the right part of Fig. 8 .

Fig. 11 shows a 3D plot of the deformed CTC model colored according to total deformation. To compare effect of different paper species on radial deformation a deformation probe on the innermost strand was placed, Fig. 11.

In Fig. 12 the maximum deformation in inwards radial direction of this probe is shown. Due to logarithmic scale of the $y$-axis the linear elastic range is logarithmical shaped. If pressure $p_{\mathrm{EL}}$ is increased the CTC suddenly collapses what results in a dramatic increase of deformation. Due to FEA convergence problems after running over this point of instability simulation was stopped. However the simulation results are sufficient to evaluate the influence of different paper aging states on radial bending stiffness.

The critical pressure $p_{\text {EL,crit }}$ a the point of instability is summarized in Table 3: For both copper species the new paper results in an increase by about $45 \%$ compared to a CTC without paper wrapping. The use of "aged1" paper with its lower plastic strain curve but the same modulus of elasticity as the new paper has no effect on critical point. The plot of maximum equivalent stress in all paper layers (Fig. 13) reveals that both new paper and "aged1" paper have the same curve. So the "aged1" paper will experience the point of damage earlier as its plastic stress strain curve ends with a lower breaking stress of about $40 \mathrm{MPa}$ compared to the new paper with about $80 \mathrm{MPa}$.

The "aged2" paper with bisected modulus of elasticity leads to a decrease of $p_{\text {EL,crit }}$ compared to the "aged1" paper. So mainly the linear part of paper stress strain curve is responsible for rigidity of the CTC model.

The plot maximum equivalent stress of all strands in Fig. 14 shows how all three paper models delay the sudden increase of mechanical stress in the strands. Point of instability is shifted to higher pressure $p_{\mathrm{EL}}$ what is equal to an increase in radial bending stiffness of CTC. 


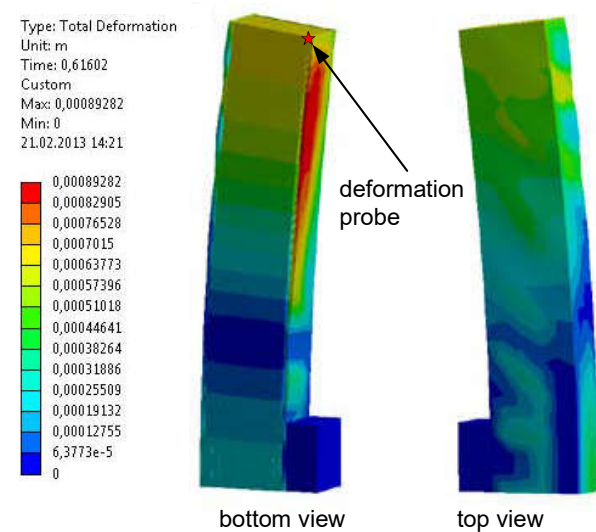

Fig. 11: 3D color plot of CTC model with copper $150 \mathrm{MPa}$, "aged2" paper at pEL $=91.5 \mathrm{kPa}$

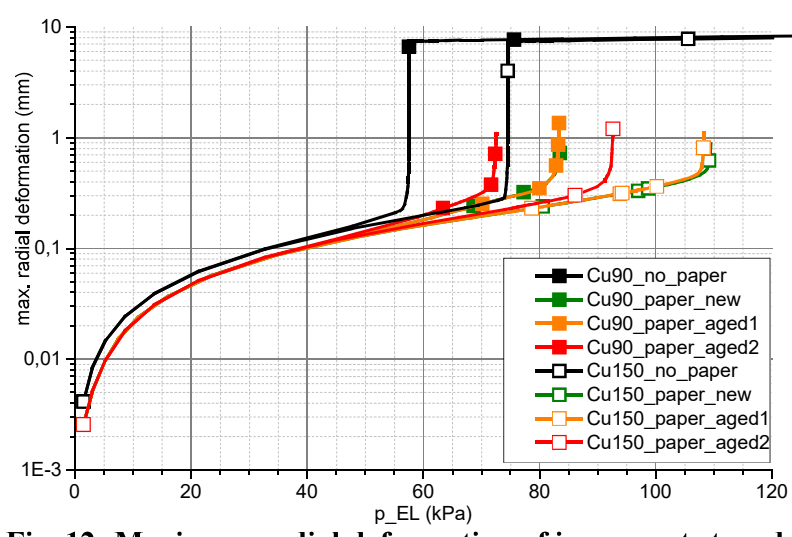

Fig. 12: Maximum radial deformation of innermost strand

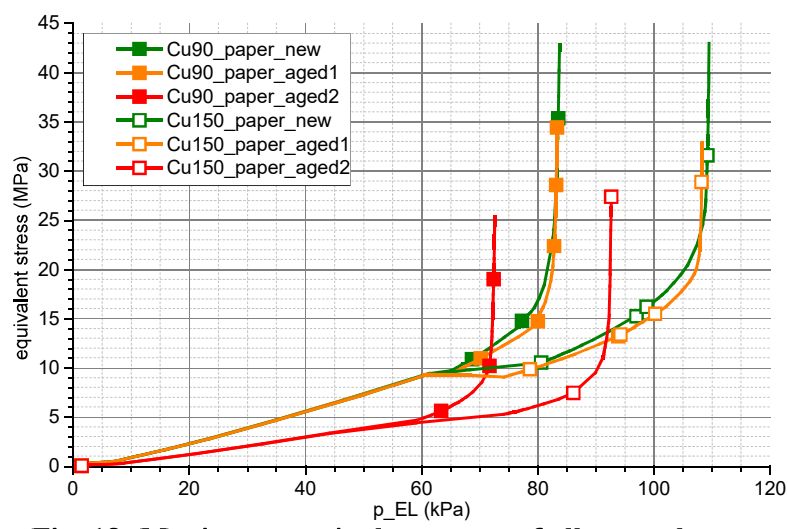

Fig. 13: Maximum equivalent stress of all paper layers

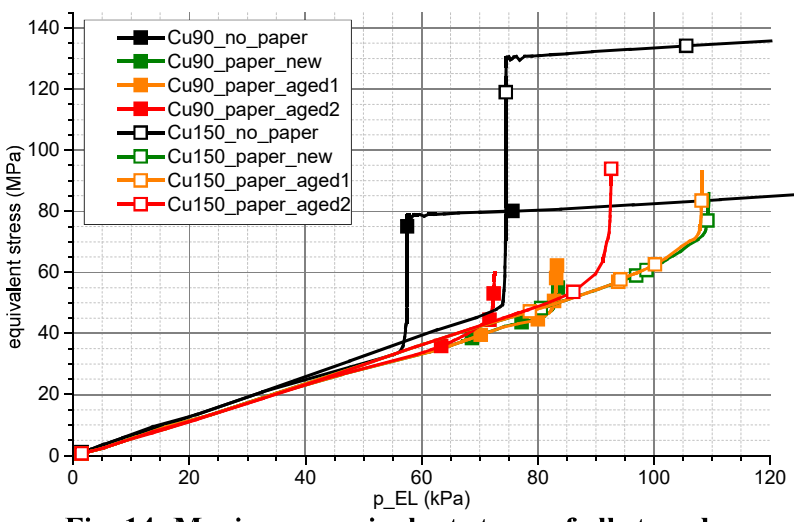

Fig. 14: Maximum equivalent stress of all strands
Table 3: critical pressure $p_{\mathrm{EL}_{\text {o }} \text {,rit }}$ at point of instability

\begin{tabular}{lrrr}
\hline copper & paper & $\boldsymbol{p}_{\text {EL,crit }}(\mathbf{k P a})$ & $\boldsymbol{p}_{\text {EL,crit }}(\mathbf{\%})$ \\
\hline Cu90 & no & 58 & 100 \\
& new & 83 & 143,1 \\
& aged1 & 83 & 143,1 \\
& aged2 & 72 & 124,1 \\
\hline Cu150 & no & 74 & 100 \\
& new & 109 & 147,3 \\
& aged1 & 108 & 145,9 \\
& aged2 & 93 & 125,6 \\
\hline
\end{tabular}

\section{Conclusion}

Two methods of evaluating the influence of paper insulation on the radial bending stiffness of CTC were proposed. The first analytic approach only respects linear material properties for a CTC segment between to axial spacers. Curvature is neglected as well as the spiral paper wrapping. It was shown that the influence of paper increases with number of single strands in radial direction and total paper thickness.

The second FEA based approach respects nonlinear material behavior and the curvature of the CTC segment. Spiral paper wrapping still remains neglected. Simulation results show that point of instability is increased for the CTC by the paper insulation. Mainly the linear modulus of elasticity of paper is responsible for CTC rigidity if thickness and number of layers remain constant. An aged paper model with decreased plastic stress strain data shows no significant difference compared to the new paper model.

A direct comparison of results from analytic and FEA approach is not possible. The analytic approach enables a quick estimation of influence from geometric dimensions while the FEA model additionally respects nonlinear material behavior and thus provides more detailed results.

\section{References}

[1] M. Faridi, V. Nabaei, S.A. Mousavi, and M. Mohammadi, "Modeling of Continuously Transposed Cable in power transformer for fault analysis based on FEM", Proc. Int. Conf. Electrical Machines and Systems, ICEMS, 2009, pp. 1-4.

[2] S. Timoshenko, "Strength of Materials, Part 1 and Part 2", D. Van Nostrand Co., Inc., Toronto New York London, 2nd Edition, 1940.

[3] ANSYS, Inc., "ANSYS Mechanical APDL Element Reference", SAS IP Inc., USA, Release 14.0, November 2011. 